\title{
AN INTERLACING THEOREM FOR MATRICES WHOSE GRAPH IS A GIVEN TREE
}

\author{
C. M. da Fonseca
}

UDC 512.643

\begin{abstract}
Let $A$ and $B$ be $(n \times n)$-matrices. For an index set $S \subset\{1, \ldots, n\}$, denote by $A(S)$ the principal submatrix that lies in the rows and columns indexed by $S$. Denote by $S^{\prime}$ the complement of $S$ and define $\eta(A, B)=\sum_{S} \operatorname{det} A(S) \operatorname{det} B\left(S^{\prime}\right)$, where the summation is over all subsets of $\{1, \ldots, n\}$ and, by convention, $\operatorname{det} A(\varnothing)=\operatorname{det} B(\varnothing)=1$. C. R. Johnson conjectured that if $A$ and $B$ are Hermitian and $A$ is positive semidefinite, then the polynomial $\eta(\lambda A,-B)$ has only real roots. G. Rublein and $\mathrm{R}$. B. Bapat proved that this is true for $n \leqslant 3$. Bapat also proved this result for any $n$ with the condition that both $A$ and $B$ are tridiagonal. In this paper, we generalize some little-known results concerning the characteristic polynomials and adjacency matrices of trees to matrices whose graph is a given tree and prove the conjecture for any $n$ under the additional assumption that both $A$ and $B$ are matrices whose graph is a tree.
\end{abstract}

\section{Introduction}

Throughout the paper, let us assume that $A=\left(a_{i j}\right)$ and $B=\left(b_{i j}\right)$ are square matrices of order $n$. For an index set $S \subset\{1, \ldots, n\}$, we denote by $A(S)$ the $|S| \times|S|$ principal submatrix lying in the rows and columns indicated by $S$. We may also write $A_{S}$ or $A[S]$ for $A\left(S^{\prime}\right)$, where $S^{\prime}$ is the complement of $S$.

Define

$$
\eta(A, B)=\sum_{S} \operatorname{det} A(S) \operatorname{det} B\left(S^{\prime}\right)
$$

where the summation is over all subsets of $\{1, \ldots, n\}$ and, by convention, $\operatorname{det} A(\varnothing)=\operatorname{det} B(\varnothing)=1$. Equivalently, considering

$$
\alpha_{k}(A, B)=\sum_{|S|=k} \operatorname{det} A(S) \operatorname{det} B\left(S^{\prime}\right)
$$

we may define

$$
\eta(A, B)=\sum_{k=0}^{n} \alpha_{k}(A, B)
$$

Note that

$$
\eta\left(\lambda I_{n},-B\right)=\operatorname{det}\left(\lambda I_{n}-B\right)
$$

i.e., $\eta\left(\lambda I_{n},-B\right)$ is the characteristic polynomial of $B$. It is well known that if $B$ is Hermitian, then the roots of (1.2), i.e., the eigenvalues of $B$, are all real. Motivated by this result, C. R. Johnson [7] considered the polynomial

$$
\eta(\lambda A,-B)=\sum_{k=0}^{n}(-1)^{n-k} \alpha_{k}(A, B) \lambda^{k}
$$

and stated the following conjecture.

Conjecture 1.1 (C. R. Johnson [7]). If $A$ and $B$ are Hermitian matrices and $A$ is positive semidefinite, then the polynomial $\eta(\lambda A,-B)$ has only real roots.

Translated from Fundamentalnaya i Prikladnaya Matematika, Vol. 10, No. 3, pp. 245-254, 2004. 
Note that if $A$ is not positive semidefinite, then the above conjecture is false. In fact, consider the matrix

$$
A=\left(\begin{array}{ll}
0 & 1 \\
1 & 0
\end{array}\right)
$$

This matrix is obviously not positive semidefinite. Setting

$$
B=\left(\begin{array}{ll}
-1 & i \\
-i & 1
\end{array}\right)
$$

we have

$$
\eta(\lambda A,-B)=-2-\lambda^{2}
$$

with pure imaginary roots

$$
\lambda_{1}=i \sqrt{2}, \quad \lambda_{2}=-i \sqrt{2} .
$$

If all roots $\lambda_{\ell}^{A}(B), \ell=1, \ldots, n$, of the polynomial (1.3) are real, then we assume that they have been arranged in increasing order:

$$
\lambda_{1}^{A}(B) \leqslant \ldots \leqslant \lambda_{n}^{A}(B)
$$

Considering the so-called Cauchy interlacing inequalities for the eigenvalues of principal submatrices of Hermitian matrices (cf., e.g., [3]), R. B. Bapat in [1] and C. R. Johnson in [8] conjectured the following.

Conjecture 1.2. If $A$ and $B$ are Hermitian matrices and $A$ is positive definite, then $\lambda_{\ell}^{A_{1}}\left(B_{1}\right)$, for $\ell=$ $1, \ldots, n-1$, interlace $\lambda_{\ell}^{A}(B)$, for $\ell=1, \ldots, n$, i.e.,

$$
\lambda_{\ell}^{A}(B) \leqslant \lambda_{\ell}^{A_{1}}\left(B_{1}\right) \leqslant \lambda_{\ell+1}^{A}(B), \quad \ell=1, \ldots, n-1 .
$$

For example, let us consider the matrices

$$
A=\left(\begin{array}{cccc}
3 & i & 2 & 1-i \\
-i & 2 & 0 & 0 \\
2 & 0 & 4 & 0 \\
1+i & 0 & 0 & 5
\end{array}\right), \quad B=\left(\begin{array}{cccc}
1 & 2 & 1+i & 1 \\
2 & -2 & 0 & 0 \\
1-i & 0 & -1 & 0 \\
1 & 0 & 0 & 3 / 2
\end{array}\right)
$$

The matrix $A$ is positive definite and

$$
\eta(\lambda A,-B)=13-24 \lambda-\lambda^{2}-21 \lambda^{3}+44 \lambda^{4}
$$

has roots

$$
\lambda_{1}=-1.49421, \quad \lambda_{2}=-0.41081, \quad \lambda_{3}=0.22289, \quad \lambda_{4}=2.15941 .
$$

On the other hand,

$$
\eta\left(\lambda A_{1},-B_{1}\right)=-3-5 \lambda+38 \lambda^{2}+40 \lambda^{3}
$$

has roots

$$
\mu_{1}=-1.00000, \quad \mu_{2}=-0.25000, \quad \mu_{3}=0.30000 .
$$

Hence

$$
\lambda_{1} \leqslant \mu_{1} \leqslant \lambda_{2} \leqslant \mu_{2} \leqslant \mu_{3} \leqslant \lambda_{4} .
$$

The Cauchy interlacing inequalities lead us to a more general conjecture.

Conjecture 1.3. Let $S$ be a subset of $\{1, \ldots, n\}$ with $k$ elements. If $A$ and $B$ are Hermitian matrices and $A$ is positive definite, then $\lambda_{\ell}^{A_{S}}\left(B_{S}\right)$, for $\ell=1, \ldots, n-k$, interlace $\lambda_{\ell}^{A}(B)$, for $\ell=1, \ldots, n$, in the following way:

$$
\lambda_{\ell}^{A}(B) \leqslant \lambda_{\ell}^{A_{S}}\left(B_{S}\right) \leqslant \lambda_{\ell+k}^{A}(B), \quad \ell=1, \ldots, n-k .
$$


If Conjecture 1.2 is true, then (1.5) can be obtained by repeated application of the interlacing inequalities (1.4) and, therefore, Conjecture 1.3 is also true.

Conjecture 1.1 has been verified for the case $n=3$ by Rublein [11]. In order to obtain the result, three subcases are considered. The treatment is quite complicated, and it seems that the proof will not carry over easily to orders $n \geqslant 4$. On the other hand, Bapat in [1] gave the solution for $n \leqslant 3$ in a very concise and elegant way. As a matter of fact, in [1] we can find the only effort to solve Conjecture 1.1 (and Conjecture 1.2) for any $n$ so far, namely when both $A$ and $B$ are tridiagonal.

Several other conjectures were proposed by Johnson [7,8]. After some difficult and considerable computational experimentation, Johnson suggested that the roots of (1.3) behave qualitatively very much like the eigenvalues of $B$. For example, (1.3) has as many positive, negative, and zero roots as the inertia of $B$ would suggest. Also, the roots of $\eta(\lambda A,-B)$ seem to be the eigenvalues of some Hermitian matrix closely related to $A$ and $B$. There are also some straightforward results. For example, if $B$ is nonsingular, then all the roots of (1.3) are nonzero.

In this paper, we generalize some results on graphs (see $[2,4,9]$ ) and use some important ideas developed in [1] to prove that the conjectures are true when both $A$ and $B$ are matrices whose graph is a tree.

\section{Weighted Trees}

A graph $G$ is a pair $(V, E)$, where $V=V(G)=\{1, \ldots, n\}$ is the vertex set and the edge set $E$ is a subset of the direct product $V \times V$. We say that vertices $i$ and $j$ are adjacent, and write $i \sim j$, if $(i, j)$ is an edge of $G$ and $i \neq j$. The symbol $\simeq$ means adjacent or equal.

A tree is a connected graph without cycles, and a forest is a graph each of whose components is a tree. In this paper, we consider finite graphs possibly containing loops (i.e., $(i, i)$ may be an edge). If to each edge $(i, j)$ a complex number is assigned, then we have a weighted graph.

If $A=\left(a_{i j}\right)$ is a Hermitian matrix, then the (weighted) graph $G=G(A)$ of $A$ is determined entirely by the off-diagonal entries of $A$ : the vertex set of $G(A)$ is $\{1, \ldots, n\}$, and $i$ and $j$ are adjacent if and only if $a_{i j} \neq 0$. The matrix $A$ is something like the weighted adjacency matrix of $G(A)$. If $A$ is a 01-matrix, with the main diagonal equal to zero, then $A$ is the adjacency matrix of $G$. In general, we consider matrices whose graph is subordinated to a tree $T$, i.e., the graph of the matrix is a subgraph of $T$.

If $S$ is a subset of $V(G)$, then $G \backslash S$ is the subgraph of $G$ induced by the vertices not in $S$. The (weighted adjacency) matrix of $G \backslash S$ is $A_{S}$. If $e=(i, j)$ is an edge of $G$, then $G \backslash e$ is obtained by deleting $e$ but not the vertices $i$ or $j$. In this case, the matrix of $G \backslash e$ is equal to that of $G$, except for the $(i, j)$-entry and, by symmetry, the $(j, i)$-entry, which are zero. Finally, $\varphi(G, \lambda)$, or simply $\varphi(G)$, is the characteristic polynomial of $A(G)$, i.e.,

$$
\varphi(G, \lambda)=\varphi(G)=\operatorname{det}\left(\lambda I_{n}-A(G)\right) .
$$

Let us define $w_{i j}(A)=-\left|a_{i j}\right|^{2}$ if $i \neq j$ and $w_{i i}(A)=a_{i i}$. Sometimes we abbreviate to $w_{i j}$. The next result provides a general recurrence relation between different characteristic polynomials.

Lemma 2.1. If $e=(i, j)$ is an edge in a (weighted) tree $T$, then

$$
\varphi(T)=\varphi(T \backslash e)+w_{i j} \varphi(T \backslash i j) .
$$

Proof. Let $E_{i j}$ be the matrix with $(i, j)$-entry equal to 1 and all other entries equal to zero. Denote by $E$ the sum $a_{i j} E_{i j}+\bar{a}_{i j} E_{j i}$. Note that

$$
A(T)=A(T \backslash e)+E .
$$

Since the determinant is a multilinear function on the columns and $T$ is a tree, we get (2.1). 
Lemma 2.2. If $T$ is a (weighted) tree and $k \in V(T)$, then

$$
\varphi(T)=\left(\lambda-w_{k k}\right) \varphi(T \backslash k)+\sum_{i \sim k} w_{k i} \varphi(T \backslash k i) .
$$

Proof. Equality (2.2) can be derived by iterating formula (2.1).

From Lemma 2.2 we can get an expression for the determinant of a tree.

Corollary 2.3. If $T$ is a (weighted) tree and $k \in V(T)$, then

$$
\operatorname{det}(T)=\sum_{i \simeq k} w_{k i} \operatorname{det}(T \backslash k i) .
$$

Proof. Set $\lambda=0$ in (2.2).

\section{An Interlacing Theorem for Trees}

For a square matrix $A$, we write $A>0$ to denote that $A$ is positive definite.

Bapat proved the veracity of Conjectures 1.1 and 1.2 in the case $n=2$.

Lemma 3.1 (R. B. Bapat [1]). Let $A$ and $B$ be Hermitian matrices of order 2 with $A>0$. Then $\eta(\lambda A,-B)$ has two real roots, say $\alpha_{1} \leqslant \alpha_{2}$. Furthermore, $\alpha_{1} \leqslant b_{22} / a_{22} \leqslant \alpha_{2}$, and the inequalities are strict if $b_{12} \neq 0$.

Note that if $b_{12}=0$, then $G(B)$ is the forest with two vertices.

The key tool to proving the main result is the following lemma.

Lemma 3.2. Let $A$ and $B$ be Hermitian matrices whose graph is a given tree. Then

$$
\eta(A, B)=\sum_{i \simeq 1}\left(w_{1 i}(A)+w_{1 i}(B)\right) \eta\left(A_{1 i}, B_{1 i}\right) .
$$

Proof. Let $C=\{i \mid i \sim 1\}$. For each subset $P$ of $C$ consider

$$
\mathcal{A}_{P}=\left\{S \mid 1 \in S, P \subset S, \text { and } P^{\prime} \cap S=\varnothing\right\}
$$

and

$$
\mathcal{C}_{P}=\left\{S \mid 1 \notin S, P \subset S, \text { and } P^{\prime} \cap S=\varnothing\right\},
$$

where $P^{\prime}$ is the complement of $P$ with respect to $C$. Thus, we get a set of parts of $\{1, \ldots, n\}$. For $S \in \mathcal{A}_{P}$, we have

$$
\operatorname{det} A(S)=\sum_{i \simeq k} w_{k i} \operatorname{det} A(S \backslash k i)
$$

and for $S \in \mathcal{C}_{P}$, we have

$$
\operatorname{det} B\left(S^{\prime}\right)=\sum_{i \simeq k} w_{k i} \operatorname{det} B\left(S^{\prime} \backslash k i\right) .
$$

Substituting all these expressions in (1.1), we get the result.

Remark 3.1. Formula (3.1) can be generalized to any vertex $k$ in the following way:

$$
\eta(A, B)=\sum_{i \simeq k}\left(w_{k i}(A)+w_{k i}(B)\right) \eta\left(A_{k i}, B_{k i}\right) .
$$

Using now induction on $n$, we can follow the proof of Theorem 3 in [1] (included here for completeness) and get the main result. 
Theorem 3.3. Let $A$ and $B$ be Hermitian matrices whose graph is a given tree, and let $A>0$. Then $\eta(\lambda A,-B)$ has real roots, say

Furthermore, if

$$
\lambda_{1} \leqslant \lambda_{2} \leqslant \ldots \leqslant \lambda_{n}
$$

are the roots of $\eta\left(\lambda A_{1},-B_{1}\right)$, then

$$
\mu_{1} \leqslant \mu_{2} \leqslant \ldots \leqslant \mu_{n-1}
$$

$$
\lambda_{1} \leqslant \mu_{1} \leqslant \lambda_{2} \leqslant \mu_{2} \leqslant \ldots \leqslant \mu_{n-1} \leqslant \lambda_{n} .
$$

Proof. For $n=1$ and $n=2$ the result is already known. Suppose now that the result is true for matrices of order $n-1$. Let us prove that the same is true for $n$.

By (3.1),

$$
\eta(\lambda A,-B)=\left(\lambda a_{11}-b_{11}\right) \eta\left(\lambda A_{1},-B_{1}\right)-\sum_{i \sim 1}\left(\lambda^{2}\left|a_{1 i}\right|^{2}+\left|b_{1 i}\right|^{2}\right) \eta\left(\lambda A_{1 i},-B_{1 i}\right) .
$$

By hypothesis, the roots $\mu_{1}, \mu_{2}, \ldots, \mu_{n-1}$ of $\eta\left(\lambda A_{1},-B_{1}\right)$ and the roots $\nu_{1}^{i}, \nu_{2}^{i}, \ldots, \nu_{n-2}^{i}$ of $\eta\left(\lambda A_{1 i},-B_{1 i}\right)$, for $i \sim 1$, are real, and

$$
\mu_{1} \leqslant \nu_{1}^{i} \leqslant \mu_{2} \leqslant \ldots \leqslant \nu_{n-2}^{i} \leqslant \mu_{n-1} \text { for } i \sim 1
$$

Since $\eta\left(\lambda A_{1 i},-B_{1 i}\right) \rightarrow \infty$ as $\lambda \rightarrow \infty$, for $i \sim 1$,

$$
\operatorname{sign} \eta\left(\mu_{\ell} \lambda A_{1 i},-B_{1 i}\right)=(-)^{n-\ell-1}, \text { for } i \sim 1 \text { and for } \ell=1, \ldots, n-1 \text {. }
$$

Replacing in (3.4) $\lambda$ by $\mu_{\ell}$, we get

$$
\operatorname{sign} \eta\left(\mu_{\ell} A,-B\right)=-\sum_{i \sim 1} \operatorname{sign} \eta\left(\mu_{\ell} A_{1 i},-B_{1 i}\right)=(-)^{n-\ell}, \text { for } \ell=1, \ldots, n-1 .
$$

Finally, since $\eta(\lambda A,-B) \rightarrow( \pm)^{n} \infty$ as $\lambda \rightarrow \pm \infty$, it follows that $\eta(\lambda A,-B)$ has a root in each of the intervals

$$
\left(-\infty, \mu_{1}\right),\left(\mu_{2}, \mu_{3}\right), \ldots,\left(\mu_{n-2}, \mu_{n-1}\right),\left(\mu_{n-1}, \infty\right),
$$

and therefore $\eta(\lambda A,-B)$ has $n$ real roots which interlace $\mu_{1}, \mu_{2}, \ldots, \mu_{n-1}$.

In [1], Bapat considered tridiagonal Hermitian matrices, i.e., Hermitian matrices whose graph is a given path, which is a special example of a tree.

The graph of the matrices $A$ and $B$ from the second example of the initial section is the star with 4 vertices:

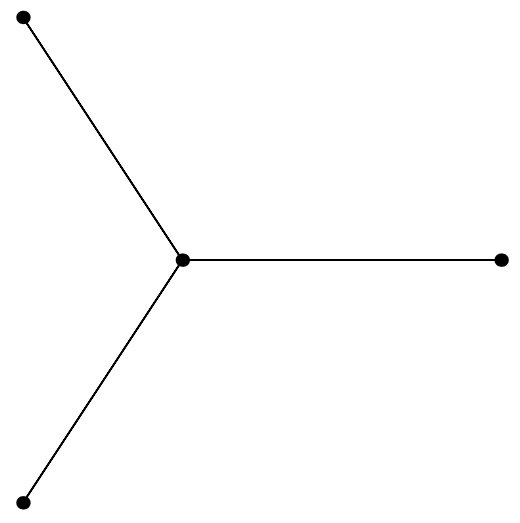

Fig. 1. Star with 4 vertices.

We remark that Theorem 3.3 is also true for the roots of $\eta\left(\lambda A_{\ell},-B_{\ell}\right)$, i.e., the roots of $\eta\left(\lambda A_{\ell},-B_{\ell}\right)$ interlace those of $\eta(\lambda A,-B)$ (see $[8,(3)])$. 


\section{Generalizations and Consequences}

If we cut a set of vertices of the graphs of $A$ and $B$, then we get some generalizations of previous results.

Theorem 4.1. Let $A$ and $B$ be Hermitian matrices whose graph is a given tree, let $A>0$, and let $S$ be a subset of $V(G)$ such that $|S|=k \geqslant 1$. Then the $n$ real roots of $\eta(\lambda A,-B)$, say $\lambda_{1} \leqslant \lambda_{2} \leqslant \ldots \leqslant \lambda_{n}$, and the $n-k$ real roots of $\eta\left(\lambda A_{S},-B_{S}\right)$, say $\mu_{1} \leqslant \mu_{2} \leqslant \ldots \leqslant \mu_{n-k}$, interlace as follows:

$$
\lambda_{\ell} \leqslant \mu_{\ell} \leqslant \lambda_{\ell+k}, \quad \ell=1, \ldots, n-k .
$$

Proof. Inequalities (4.1) can be obtained by repeated application of the interlacing inequalities (3.3) from Theorem 3.3.

Consider the following tree with 5 vertices.

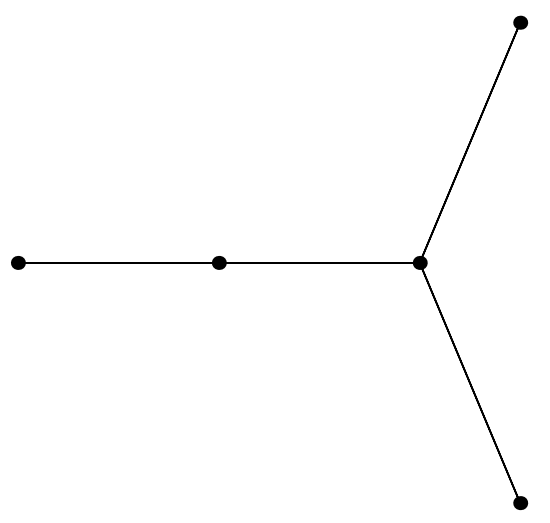

Fig. 2. A tree with 5 vertices.

Let us consider the Hermitian positive-definite matrix whose graph is the tree from Fig. 2:

$$
A=\left(\begin{array}{ccccc}
2 & 1 / 2 & 0 & 0 & 0 \\
1 / 2 & 1 & -1 & 0 & 0 \\
0 & -1 & 5 & -2 & -1+i \\
0 & 0 & -2 & 3 & 0 \\
0 & 0 & -1-i & 0 & 4
\end{array}\right) .
$$

The graph of the matrix

is the same tree. We have

$$
B=\left(\begin{array}{ccccc}
0 & 2 & 0 & 0 & 0 \\
2 & -1 & 1 & 0 & 0 \\
0 & 1 & 0 & -i & -1 \\
0 & 0 & i & -1 & 0 \\
0 & 0 & -1 & 0 & 1
\end{array}\right)
$$

$$
\eta(\lambda A,-B)=50 \lambda-54 \lambda^{2}-181 \lambda^{3}+\frac{345}{4} \lambda^{4}+\frac{85}{2} \lambda^{5},
$$

with roots

$$
\lambda_{1}=-3.20024, \quad \lambda_{2}=-0.61837, \quad \lambda_{3}=0.00000, \quad \lambda_{4}=0.44093, \quad \lambda_{5}=1.34827 .
$$

Setting $S=\{2,4\}$, we get

$$
\eta\left(\lambda A_{S},-B_{S}\right)=-2 \lambda-10 \lambda^{2}+36 \lambda^{3},
$$


with roots

$$
\mu_{1}=-0.13469, \quad \mu_{2}=0.00000, \quad \mu_{3}=0.41246
$$

Therefore,

$$
\lambda_{1} \leqslant \mu_{1} \leqslant \lambda_{3}, \quad \lambda_{2} \leqslant \mu_{2} \leqslant \lambda_{4}, \quad \lambda_{3} \leqslant \mu_{3} \leqslant \lambda_{5} .
$$

Since for a Hermitian matrix $A$ such that its graph is a given forest and $A>0$ the polynomial $\eta(\lambda A,-A)$ has only real zeros, we see that the average Fischer terms

$$
\beta_{k}=\frac{\alpha_{k}(A, A)}{\left(\begin{array}{l}
n \\
k
\end{array}\right)},
$$

for $k=1, \ldots, n$, form a log-concave sequence [5, Lemma 3.2], i.e., $\beta_{k}^{2} \geqslant \beta_{k-1} \beta_{k+1}$, for $k \geqslant 1$. Therefore,

$$
\frac{\beta_{1}}{\beta_{0}} \geqslant \frac{\beta_{2}}{\beta_{1}} \geqslant \ldots \geqslant \frac{\beta_{n}}{\beta_{n-1}} .
$$

This also generalizes the results of Bapat to a larger class of matrices.

Finally, we give an analog for the Schur's result that states that if $B$ is a Hermitian matrix, then the eigenvalues of $B$ majorize the diagonal entries.

For $x, y \in \mathbb{R}^{n}$, we say that $x$ is weakly majorized by $y$, and write $x \prec_{w} y$, whenever

$$
x_{1}^{\prime}+\cdots+x_{k}^{\prime} \leqslant y_{1}^{\prime}+\cdots+y_{k}^{\prime}, \text { for all } k \in\{1, \ldots, n\},
$$

where $z_{1}^{\prime}, \ldots, z_{k}^{\prime}$ denotes the nonincreasing rearrangement of $z \in \mathbb{R}^{n}$ (see [10]).

Bapat considered tridiagonal matrices.

Theorem $4.2([1])$. Let $A$ and $B$ be positive-definite, tridiagonal matrices. Then

$$
\left(\frac{b_{11}}{a_{11}}, \ldots, \frac{b_{n n}}{a_{n n}}\right) \prec_{w}\left(\lambda_{1}^{A}(B), \ldots, \lambda_{n}^{A}(B)\right) .
$$

Taking into account the proof of Theorem 3.3, we can state the following theorem.

Theorem 4.3. Let $A$ and $B$ be Hermitian matrices whose graph is a given tree, and let $A>0$. Then

$$
\left(\frac{b_{11}}{a_{11}}, \ldots, \frac{b_{n n}}{a_{n n}}\right)
$$

is weakly majorized by

$$
\left(\lambda_{1}^{A}(B), \ldots, \lambda_{n}^{A}(B)\right)
$$

This work was supported by CMUC - Centro de Matemática da Universidade Coimbra.

\section{REFERENCES}

1. R. B. Bapat, "An interlacing theorem for tridiagonal matrices," Linear Algebra Appl., 150, 331-340 (1991).

2. D. M. Cvetković, M. Doob, and H. Sachs, Spectra of Graphs, Academic Press, New York (1980).

3. Ky Fan and G. Pall, "Imbedding conditions for Hermitian and normal matrices," Canad. J. Math., 9, 298-304 (1957).

4. G. Godsil, "Spectra of trees," Ann. Discrete Math., 20, 151-159 (1984).

5. G. Godsil, Algebraic Combinatorics, Chapman and Hall, New York (1993).

6. R. A. Horn and C. R. Johnson, Matrix Analysis, Cambridge University Press (1985).

7. C. R. Johnson, "The permanent-on-top conjecture: A status report," in: F. Uhlig and R. Grone, eds., Current Trends in Matrix Theory, Elsevier Science (1987), pp. 167-174.

8. C. R. Johnson, "A characteristic polynomial for matrix pairs," Linear and Multilinear Algebra, 25, 289-290 (1989).

9. L. Lovász, Combinatorial Problems and Exercises, North-Holland, Amsterdam (1979). 
10. A. W. Marshall and I. Olkin, Inequalities: Theory of Majorization and Its Applications, Academic Press, New York (1976).

11. G. Rublein, "On a conjecture of C. Johnson," Linear and Multilinear Algebra, 25, 257-267 (1989).

C. M. da Fonseca

Departamento de Matemática, Universidade de Coimbra, 3001-454 Coimbra, Portugal

E-mail: cmf@mat.uc.pt 